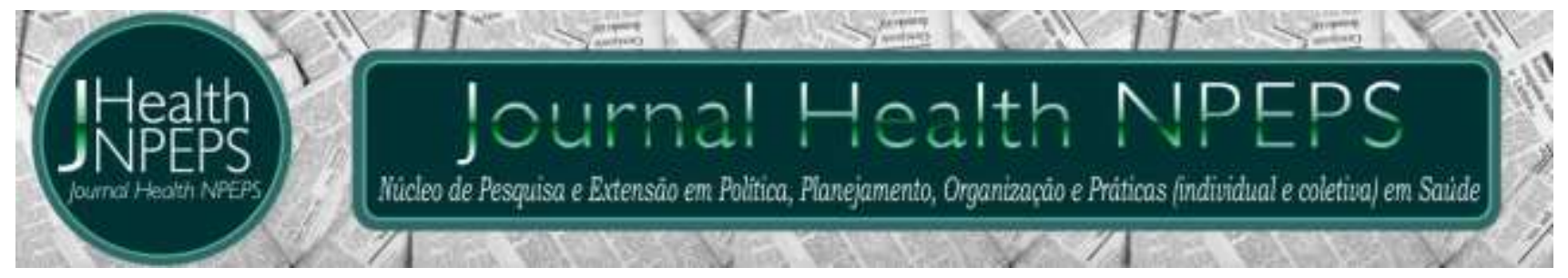

http://dx.doi.org/10.30681/252610104697

ARTIGO ORIGINAL

\title{
Análise descritiva dos casos confirmados de leptospirose em humanos no Brasil, período de 2010-2019
}

\section{Descriptive analysis of confirmed cases of leptospirosis in humans in Brazil from 2010-2019}

\section{Análisis descriptivo de casos confirmados de leptospirosis en humanos em Brasil de 2010-2019}

\author{
Flávio Aparecido da Cruz Magalhães ${ }^{1}$, Rayniel de Moraes Mendes², \\ Andréia Lima Tomé Melo ${ }^{3}$
}

\section{RESUMO}

Objetivo: descrever o número populacional de casos confirmados de leptospirose em humanos no Brasil no período de 2010 a 2019. Método: estudo transversal, pautado em dados secundários, disponíveis no Sistema de Informação de Agravos de Notificação. As variáveis consideradas foram número de casos confirmados por Unidade da Federação, capital, mês, ano de notificação, sexo, faixa etária, etnia/cor, escolaridade, área/local de infecção, ambiente de infecção, critério de confirmação e evolução do agravo. Optou-se por calcular a incidência média por Unidade da Federação e capitais, e os dados foram apresentados de forma agrupada. Resultados: em relação aos estados, oito se destacaram pela alta incidência. Em relação ao sexo, o predomínio foi de homens (79,6\%), na faixa etária de 20 a 59 anos $(72,4 \%)$, especificamente aqueles entre 20 e 39 anos $(39,6 \%)$, brancos $(44,8 \%)$, com baixo nível de escolaridade $(58,9 \%)$. A ocorrência dos casos foi em região urbana $(54,9 \%)$ e contágio domiciliar $(41 \%)$. Em relação ao acesso aos serviços de saúde, diagnóstico e tratamento, prevaleceu diagnóstico clínico laboratorial $(86,8 \%)$, com a maioria dos casos evoluindo para a cura $(83,1 \%)$. Conclusão: a leptospirose apresenta um comportamento endêmico no Brasil.

Descritores: Leptospira; Humanos; Zoonoses.

\section{ABSTRACT}

Objective: to describe the number of confirmed cases of leptospirosis in humans in Brazil from 2010 to 2019. Method: cross-sectional study based on secondary data,

\footnotetext{
${ }^{1}$ Biomédico. Doutorando do Programa de Pós-Graduação em Biociência Animal da Universidade de Cuiabá (UNIC). Docente e Coordenador do Curso de Graduação em Biomedicina da Faculdade Fasipe CPA (Cuiabá). Cuiabá, Mato Grosso, Brasil. E-mail: fbiomedicas@gmail.com ORCID ID: http://orcid.org/0000-0003-0305-4138 Autor para correspondência - Endereço: Rodovia Mario Andreaza, 399 - Varzea grande MT. Cep.: 78.144-902. Brasil.

${ }^{2}$ Acadêmico de Biomedicina pela Universidade de Cuiabá (UNIC). Cuiabá, Mato Grosso, Brasil. E-mail: raynielbiomed@gmail.com ORCID ID: http://orcid.org/0000-003-0206-0413

${ }_{3}^{3}$ Médica Veterinária. Doutora em Ciências Veterinárias. Docente do Curso de Graduação em Medicina Veterinária. Universidade de Cuiabá (UNIC). Cuiabá, Mato Grosso, Brasil. E-mail: andreialtm@gmail.com ORCID ID: http://orcid.org/0000-0001-7329-4255
} 
available in the Notifiable Diseases Information System. The variables considered were number of cases confirmed by Federation Unit, capital, month, year of notification, gender, age group, ethnicity/color, education, area/place of infection, infection environment, confirmation criteria and disease evolution. It was decided to calculate the average incidence by Federation Unit and capitals, and the data were presented in a grouped way. Results: in relation to the states, eight stood out for their high incidence. Regarding gender, the predominance was of men (79.6\%), aged 20 to 59 years (72.4\%), specifically those between 20 and 39 years (39.6\%), white (44.8\%), with low level of education (58.9\%). The occurrence of cases was in an urban region (54.9\%) and home contagion (41\%). Regarding access to health services, diagnosis and treatment, laboratory clinical diagnosis (86.8\%) prevailed, with most cases progressing to cure (83.1\%). Conclusion: leptospirosis presents an endemic behavior in Brazil.

Descriptors: Leptospira; Humans; Zoonoses.

\section{RESUMEN}

Objetivo: describir el número de casos confirmados de leptospirosis en humanos en Brasil de 2010 a 2019. Método: estudio transversal basado en datos secundarios, disponible en el Sistema de Información sobre Enfermedades Notificable. Las variables consideradas fueron el número de casos confirmados por la Unidad de la Federación, capital, mes, año de notificación, género, grupo de edad, etnia/color, educación, áreal lugar de infección, entorno de infección, criterios de confirmación y evolución de la enfermedad. Se decidió calcular la incidencia media por Unidad de Federación y capitales, y los datos se presentaron de forma agrupada. Resultados: en relación con los estados, ocho destacaron por su alta incidencia. En cuanto al género, el predominio fue el de los hombres (79,6\%), de 20 a 59 años (72,4\%), concretamente los de entre 20 y 39 años $(39,6 \%)$, blancos $(44,8 \%)$, con bajo nivel educativo $(58,9 \%)$. La ocurrencia de casos se registró en una región urbana (54,9\%) y contagio en el hogar (41\%). En cuanto al acceso a los servicios de salud, diagnóstico y tratamiento, prevaleció el diagnóstico clínico de laboratorio (86,8\%), con la mayoría de los casos progresando en curación $(83,1 \%)$. Conclusión: la leptospirosis presenta un comportamiento endémico en Brasil.

Descriptores: Leptospira; Humanos; Zoonosis.

\section{INTRODUÇÃO}

A leptospirose é uma zoonose infecciosa aguda, presente em locais de clima tropical e subtropical, causada por bactérias espiraladas do gênero Leptospira e cuja sua ocorrência está relacionada a diversos fatores. Dentre esses fatores, destaca-se aspectos socioeconômicos, ambientais e sanitários ${ }^{1}$.

\section{A classificação desse gênero} bacteriano foi definida pelos Sorovares (sorotipo), o que permitiu organizar esta bactéria em mais de 200 sorovares diferentes. Clinicamente

a sintomatologia é muito variada, desde casos leves até casos severos com agressão hepática e renal significativas. A transmissão se dá principalmente pela urina de animais infectados como bovinos, suínos, caprinos, cães, gatos e ratos de esgoto ${ }^{2,3}$. Nos humanos a 
transmissão ocorre de forma acidental, devido ao contato com urina de animais infectados ou mesmo com água, desde que estejam contaminadas com a bactéria $^{4-7}$.

A doença está presente em vários países, especialmente aqueles com predominância dos climas tropical e subtropical, como aqueles da América do Sul, a exemplo do Brasil3,8. Estima-se que no mundo ocorra anualmente cerca de 1 milhão de novos casos e cerca de 58.900 mortes por leptospirose. Já na América Latina, tem-se que a estimativa da mortalidade anual seja de 3,9 por 100.000 habitantes?.

No Brasil, as pesquisas apontam que a ocorrência da leptospirose possui relação com condições inadequadas de habitação, saneamento básico precário, ausência de rede de esgoto e dificuldade de acesso à água tratada ${ }^{1}$. Grande parte desta população, encontra-se em estado de grande vulnerabilidade social $\mathrm{e}$ econômica, o que gera condições ambientais e comportamentais para a ocorrência de leptospirose em diversas regiões brasileiras. Diante do exposto, este estudo teve como objetivo descrever o número populacional de casos confirmados de leptospirose em humanos no Brasil no período de 2010 a 2019.

\section{MÉTODO}

Trata-se de estudo transversal e com abordagem quantitativa, pautado em dados secundários dos casos de leptospirose humana no Brasil, disponíveis no Sistema de Informação de Agravos de Notificação (SINAN), oriundos do Departamento de Informática do Sistema Único de Saúde (DATASUS) entre janeiro de 2010 a dezembro de 2019.

Os dados foram obtidos de forma eletrônica entre os meses de março a julho de 2020, pelos próprios pesquisadores. Considerou-se as variáveis, número de casos confirmados por Unidade da Federação (UF) de notificação, capital, mês e ano de notificação, sexo, faixa etária, etnia/cor, escolaridade, área/local de infecção, ambiente de infecção, critério de confirmação e evolução do agravo.

Para diminuir 0 viés de interpretação dos dados brutos, optou-se por calcular a incidência média por UF e capitais. Para tabulação dos dados foi usado o programa Microsoft Excel para Mac, versão 16.33 (20011301) 2019, com realização de análise descritiva e apresentação em tabelas.

O estudo respeitou todos os aspectos éticos em pesquisas que 
utilizaram dados públicos e abertos, de acordo com a resolução 466/2012. Todas as fontes utilizadas, foram devidamente descritas.

\section{RESULTADOS}

Foram observados 37.841 casos de leptospirose em humanos, distribuídos por todos os Estados brasileiros (Tabela 1). Os anos de maior ocorrência compreendem à 2011, 2013, 2014 e 2015, que somados correspondem a $18.234(48,18 \%)$ dos casos. Em relação aos Estados, considerando a incidência média, oito se destacaram, Rondônia $(34,12)$, Acre $(438,25)$, Amapá $(85,29)$, Pernambuco $(22,00)$, Espírito Santo $(39,03)$, Paraná $(30,33)$, Santa Catarina $(55,10)$ e Rio Grande do Sul $(41,78)$.

$\mathrm{Na}$ Tabela 2, é possível observar que as capitais que apresentam as maiores incidências, são Rio Branco $(75,71)$ e Macapá $(10,02)$. As demais capitais apresentaram incidência menores, entretanto, os dados indicam que a ocorrência dos casos não se concentra somente nas capitais. Quando aos meses de ocorrência, é possível observar que a distribuição ocorre praticamente o ano todo, com destaque para os meses de janeiro a julho.

Ao avaliar a Tabela 3, evidencia que a maioria dos casos ocorreu em homens $(79,6 \%)$, na faixa etária de 20 a 59 anos $(72,4 \%)$, principalmente entre os 20-39 anos.

Em relação a cor e escolaridade, foi observado predomínio de brancos $(44,8)$ e pardos $(38,2)$. No que tange a escolaridade, dos dados informados, observa-se maior ocorrência em indivíduos com menor nível de escolaridade.

Quando avalia a área/local e ambiente de infecção, tem-se maiores nas áreas urbanas, 20.768 (54,9\%), sendo maior em ambiente domiciliar, 15.514 $(41,0 \%)$ dos casos. Quanto ao critério de confirmação houve $32.863(86,8 \%)$ casos confirmados laboratorialmente e em relação a evolução do agravo, $31.461(83,1 \%)$ dos casos evoluíram para cura e $3.258(8,6 \%)$ para óbito pelo agravo. 
Tabela 1 - Incidência média e distribuição de casos confirmado notificados de leptospirose por Estados brasileiros no período de 2010 a 2019. Brasil.

\begin{tabular}{|c|c|c|c|c|c|c|c|c|c|c|c|c|}
\hline UF de notificação & 2010 & 2011 & 2012 & 2013 & 2014 & 2015 & 2016 & 2017 & 2018 & 2019 & Total & Incidência \\
\hline Rondônia & 14 & 56 & 9 & 151 & 186 & 85 & 40 & 21 & 27 & 24 & 613 & 34,12 \\
\hline Acre & 45 & 130 & 259 & 529 & 1222 & 968 & 189 & 225 & 172 & 181 & 3920 & 438,25 \\
\hline Amazonas & 38 & 75 & 72 & 62 & 82 & 76 & 45 & 72 & 59 & 46 & 627 & 14,9 \\
\hline Roraima & 2 & - & 3 & 5 & 4 & 1 & - & 2 & 2 & 2 & 21 & 3,33 \\
\hline Pará & 91 & 131 & 100 & 124 & 133 & 129 & 122 & 126 & 136 & 133 & 1225 & 14,1 \\
\hline Amapá & 67 & 98 & 82 & 49 & 88 & 52 & 81 & 68 & 84 & 66 & 735 & 85,29 \\
\hline Tocantins & 1 & 4 & 4 & 11 & 3 & 1 & 3 & 3 & 11 & 13 & 54 & 3,4 \\
\hline Maranhão & 39 & 48 & 20 & 19 & 34 & 20 & 14 & 22 & 23 & 30 & 269 & 3,78 \\
\hline Piauí & 2 & 1 & 3 & 3 & 2 & 2 & 2 & - & 6 & 21 & 42 & 1,28 \\
\hline Ceará & 37 & 134 & 60 & 31 & 50 & 30 & 48 & 28 & 52 & 105 & 575 & 6,26 \\
\hline Rio Grande do Norte & 20 & 35 & 14 & 6 & 13 & 22 & 4 & 3 & 13 & 12 & 142 & 4,02 \\
\hline Paraíba & 8 & 26 & 16 & 21 & 16 & 11 & 8 & 11 & 18 & 21 & 156 & 3,86 \\
\hline Pernambuco & 270 & 380 & 122 & 170 & 222 & 141 & 158 & 219 & 211 & 223 & 2116 & 22 \\
\hline Alagoas & 68 & 86 & 49 & 56 & 70 & 34 & 15 & 71 & 49 & 60 & 558 & 16,65 \\
\hline Sergipe & 76 & 51 & 36 & 33 & 45 & 38 & 21 & 33 & 24 & 28 & 385 & 16,6 \\
\hline Bahia & 197 & 169 & 94 & 182 & 121 & 126 & 54 & 76 & 69 & 76 & 1164 & 7,8 \\
\hline Minas Gerais & 99 & 110 & 126 & 140 & 136 & 112 & 155 & 129 & 179 & 178 & 1364 & 6,41 \\
\hline Espírito Santo & 269 & 305 & 239 & 156 & 252 & 77 & 53 & 71 & 71 & 93 & 1586 & 39,03 \\
\hline Rio de Janeiro & 295 & 422 & 186 & 232 & 167 & 137 & 164 & 165 & 242 & 213 & 2223 & 12,8 \\
\hline São Paulo & 875 & 999 & 763 & 974 & 773 & 622 & 601 & 554 & 529 & 514 & 7204 & 15,56 \\
\hline Paraná & 317 & 477 & 224 & 334 & 246 & 496 & 433 & 257 & 321 & 388 & 3493 & 30,33 \\
\hline Santa Catarina & 432 & 704 & 411 & 339 & 350 & 545 & 377 & 315 & 254 & 269 & 3996 & 55,1 \\
\hline Rio Grande do Sul & 476 & 544 & 274 & 438 & 480 & 532 & 403 & 494 & 448 & 683 & 4772 & 41,78 \\
\hline Mato Grosso do Sul & 2 & - & 6 & 4 & 18 & 13 & 9 & 8 & 9 & 9 & 78 & 2,78 \\
\hline Mato Grosso & 3 & 8 & 6 & 3 & 4 & 11 & 7 & 14 & 19 & 25 & 100 & 2,84 \\
\hline Goiás & 7 & 3 & 19 & 25 & 21 & 18 & 22 & 23 & 20 & 17 & 175 & 2,46 \\
\hline Distrito Federal & 35 & 13 & 24 & 34 & 19 & 38 & 36 & 9 & 22 & 18 & 248 & 8,12 \\
\hline Total & 3785 & 5009 & 3221 & 4131 & 4757 & 4337 & 3064 & 3019 & 3070 & 3448 & 37841 & \\
\hline
\end{tabular}

Tabela 2 - Incidência média e distribuição dos casos confirmados de leptospirose por capital de notificação e mês de notificação no período de 2010 a 2019. Brasil.

\begin{tabular}{lcccccccccccccc}
\hline $\begin{array}{l}\text { Capital de } \\
\text { notificação }\end{array}$ & Jan & Fev & Mar & Abr & Mai & Jun & Jul & $\begin{array}{c}\text { Ag } \\
\text { o }\end{array}$ & Set & $\begin{array}{c}\text { Ou } \\
\text { t }\end{array}$ & $\begin{array}{c}\text { No } \\
\text { v }\end{array}$ & $\begin{array}{c}\text { De } \\
\text { z }\end{array}$ & Total & $\begin{array}{c}\text { Incidênci } \\
\text { a }\end{array}$ \\
\hline Porto Velho & 16 & 23 & 39 & 37 & 32 & 41 & 45 & 9 & 8 & 10 & 16 & 3 & 279 & 5,17 \\
Rio Branco & 180 & 262 & 818 & 505 & 274 & 204 & 177 & 141 & $\begin{array}{c}12 \\
9\end{array}$ & 15 & 134 & 152 & 3130 & 75,71 \\
Manaus & 47 & 64 & 81 & 59 & 71 & 52 & 44 & 35 & 18 & 29 & 21 & 40 & 561 & 2,53 \\
Boa Vista & - & 2 & 1 & 3 & 1 & 2 & 4 & 2 & - & 2 & 2 & 1 & 20 & 0,48 \\
Belém & 56 & 74 & 84 & 95 & 89 & 59 & 37 & 53 & 27 & 21 & 17 & 33 & 645 & 4,30 \\
Macapá & 32 & 45 & 70 & 71 & 51 & 46 & 39 & 38 & 42 & 30 & 29 & 21 & 514 & 10,02 \\
Palmas & 2 & 2 & - & 3 & 7 & 5 & - & - & 1 & - & 1 & 2 & 23 & 0,75 \\
São Luís & 14 & 10 & 26 & 24 & 45 & 24 & 20 & 17 & 8 & 4 & 4 & 6 & 202 & 1,82 \\
Teresina & 3 & 4 & 3 & 13 & 9 & 1 & 1 & - & - & 1 & - & 1 & 36 & 0,41 \\
Fortaleza & 14 & 29 & 48 & 92 & 76 & 36 & 37 & 33 & 16 & 14 & 12 & 14 & 421 & 1,57
\end{tabular}




\begin{tabular}{lcccccccccccccc} 
Natal & 4 & 9 & 7 & 8 & 9 & 7 & 10 & 13 & 7 & 6 & 4 & 3 & 87 & 0,98 \\
João Pessoa & 7 & 6 & 16 & 7 & 18 & 8 & 13 & 12 & 13 & 1 & 6 & 2 & 109 & 1,33 \\
Recife & 46 & 79 & 69 & 105 & 221 & 158 & 333 & 204 & 99 & 38 & 52 & 39 & 1443 & 8,73 \\
Maceió & 29 & 21 & 27 & 39 & 65 & 59 & 104 & 66 & 34 & 27 & 29 & 15 & 515 & 5,02 \\
Aracaju & 21 & 10 & 20 & 39 & 30 & 58 & 54 & 32 & 30 & 14 & 22 & 7 & 337 & 5,07 \\
Salvador & 43 & 28 & 35 & 85 & 124 & 115 & 124 & 75 & 61 & 37 & 41 & 40 & 808 & 2,80 \\
Belo Horizonte & 52 & 41 & 25 & 21 & 18 & 13 & 10 & 15 & 8 & 10 & 17 & 31 & 261 & 1,04 \\
Vitória & 55 & 27 & 29 & 29 & 21 & 14 & 11 & 12 & 8 & 5 & 16 & 24 & 251 & 6,86 \\
Rio de Janeiro & 95 & 74 & 94 & 85 & 50 & 45 & 35 & 27 & 37 & 21 & 57 & 61 & 681 & 1,01 \\
São Paulo & 348 & 407 & 362 & 237 & 106 & 109 & 106 & 65 & 69 & 86 & 104 & 124 & 2123 & 1,72 \\
Curitiba & 231 & 268 & 257 & 139 & 94 & 96 & 64 & 38 & 39 & 71 & 84 & 95 & 1476 & 7,57 \\
Florianópolis & 63 & 66 & 60 & 38 & 25 & 26 & 16 & 10 & 22 & 23 & 21 & 36 & 406 & 7,98 \\
Porto Alegre & 77 & 97 & 95 & 59 & 62 & 50 & 30 & 30 & 40 & 46 & 44 & 47 & 677 & 4,55 \\
Campo Grande & 9 & 9 & 4 & 10 & 9 & - & 2 & 1 & 3 & 1 & 2 & 5 & 55 & 0,61 \\
Cuiabá & 1 & 3 & 1 & 3 & 1 & - & 1 & 1 & 3 & - & - & 1 & 15 & 0,24 \\
Goiânia & 11 & 13 & 9 & 9 & 8 & 6 & 5 & 6 & 2 & 2 & 3 & 6 & 80 & 0,52 \\
Brasília & 45 & 34 & 29 & 41 & 23 & 13 & 15 & 4 & 5 & 12 & 15 & 12 & 248 & 0,81 \\
\hline Total & 150 & 170 & 230 & 185 & 153 & 124 & 133 & 939 & 72 & 66 & 753 & 821 & 1540 & \\
\hline
\end{tabular}

Tabela 3 - Características sociodemográficas, critério de confirmação e evolução dos casos confirmados e notificados no período de 2010 a 2019. Brasil.

\begin{tabular}{lcc}
\hline Variáveis & $\mathrm{N}$ & $\%$ \\
\hline Sexo & 30.120 & 79,6 \\
Masculino & 7.721 & 20,4 \\
Feminino & & \\
Faixa Etária & 405 & 1,07 \\
$\leq$ O4 anos & 3.006 & 7,9 \\
O5 a 14 anos & 3.401 & 9,0 \\
15 a 19 anos & 14.957 & 39,5 \\
20 a 39 anos & 12.453 & 32,9 \\
40 a 59 anos & 2.586 & 6,8 \\
60 a 69 anos & 1.023 & 2,7 \\
$\geq 70$ anos & 10 & 0,1 \\
NI* & & \\
Etnia/Cor & 16.957 & 44,8 \\
Branca & 2.049 & 5,4 \\
Preta & 237 & 0,6 \\
Amarela & 14.428 & 38,2 \\
Parda/morena & 112 & 0,3 \\
Indígena & 4.058 & 10,7 \\
NI & & \\
Escolaridade & 592 & 1,6 \\
Analfabeto & 14.253 & 37,7 \\
E. Fundamental & 7.297 & 19,3 \\
E. Médio & 1.338 & 3,5 \\
E. Superior ou mais & 14.361 & 37,9 \\
NI & & \\
Área/local de infecção & 20.768 & 54,9 \\
Urbana & 8.335 & 22,0 \\
Rural & 1.194 & 3,1 \\
Periurbana & 7.544 & 20,0 \\
NI & & \\
Ambiente de infecção & &
\end{tabular}


Domiciliar

Trabalho

Lazer

Outros

NI

Critério de confirmação

Clínico-Laboratorial

Clínico-Epidemiológico

NI

Evolução

Cura

Óbito pelo agravo

Óbito por outra causa

$\mathrm{NI}$
15.514

6.563

2.483

2.409

10.872

32.863

4.566

412

31.461

3.258

322

2800
41,0

17,3

6,6

6,4

28,7

86,8

12,1

1,1

83,1

8,6

0,9

7,4

\section{DISCUSSÃO}

$\mathrm{Na}$ América Latina e no continente europeu, os casos de leptospirose humana atingem sobretudo a população com baixo poder econômico, com pouco ou nenhum acesso a saneamento básico visto que o controle da doença consiste inclusive no melhoramento do saneamento básico e das condições de habitaçãa ${ }^{10-16}$. Os resultados observados nesse estudo, reforçam os fatores socioeconômicos relacionados à ocorrência da leptospirose e corroboram à achados de outros autores ${ }^{1,17-20}$.

Ainda que os fatores relacionados à densidade populacional e organização socioeconômica da população possuam relação com a ocorrência de $\operatorname{casos}^{5}$, e que no Brasil, as regiões Sul e Sudeste sejam as responsáveis pelo maior quantitativo de casos, nesse estudo identificou que as capitais de outras regiões mesmo com baixa densidade populacional, apresentaram alta incidência de leptospirose como, por exemplo, Rio Branco (AC), possivelmente pelas características da rede de atenção à saúde, aspectos climáticos e pluviométricos da localidade. Estudo em Santa Catarina (BR), também não observou associações das taxas de incidência com os fatores demográficos, e sim com baixas altitudes, altas temperaturas e áreas de risco de desastres naturais ${ }^{21}$.

Observa-se que o período de janeiro a julho foram os meses em que houve os maiores números de casos, o que demonstra a importância das condições ambientais como um dos fatores associados à doença ${ }^{18,19,21}$. Essa relação com meses mais chuvosos, também pode estar determinando a concentração de casos em regiões próximas da Amazônia e no litoral de 
países latinos ${ }^{22,23}$. Por outro lado, Roraima (BR) que pertence a região da Amazônia brasileira foi o único Estado com ausência de vários registros de leptospirose no período investigado, o que sinaliza comportamentos variáveis da doença pelo continente.

Os casos de leptospirose em Portugal, tanto no continente como nos açores, surgem predominantemente associados à faixa etária dos 25 aos 64 anos $(60,4 \%)^{11}$. No presente estudo, a faixa etária concentrou na fase de maior produtividade profissional dos brasileiros, o que indica, ainda a ser investigado, possíveis relações do trabalho, autocuidado e acesso à educação em saúde/literacia em saúde com a maior vulnerável à leptospirose.

Em relação às características étnicas, nota-se que a população brasileira é constituída pela somatória de várias etnias, e suas relações de desigualdades ainda se mantem presentes. Embora tenha observado maior registro de pessoas brancas no período investigado, estudos apontam que pretos e pardos são mais vulneráveis e estão mais susceptíveis às situações de risco ${ }^{1}$, porém isso pode ser alterado pelas características de origem da população exposta.
Ao analisar a área/local e ambiente de infecção, observa-se que os casos se concentram na área urbana e no ambiente domiciliar, assim como em outros estudos no cenário nacional, em períodos anteriores ${ }^{24,25}$. No que se refere ao diagnóstico e evolução da doença, os dados demonstram que o acesso aos serviços de saúde, mesmo com todas as restrições, pode contribuir para melhores prognósticos ${ }^{1,21}$. Embora existam evidências na literatura que nem todos as regiões brasileiras que registram casos da doença conseguem manejar adequadamente os $\operatorname{casos}^{26}$, e um dos motivos para essa situação se deve a estreita relação do número de notificações vs investimentos em estudos e treinamentos, em consequência dificuldades na terapêutica como, diante de anormalidades de exames laboratoriais $^{27}$. No Brasil, isso já se observa facilmente ao vislumbrar que grandes editais temáticos na área de doenças negligenciadas não fazem qualquer menção à pesquisas e intervenções para a leptospirose ${ }^{5}$.

Além disso, um problema recorrente em todo mundo tanto em regiões tropicais e não tropicais, que também podem estar influenciando a incidência de casos de leptospirose são as informações limitadas, quer seja por 
procedimentos não padronizados de investigações diagnósticas como de notificação ${ }^{28}$. Como a leptospirose é um agravo de notificação compulsória desde 1987 no país, acredita-se que possíveis falhas ocorram não exclusivamente no processo de registro no SINAN, e sim entre $\mathrm{o}$ primeiro atendimento até $\mathrm{O}$ tempo transcorrido do reconhecimento de sinais/sintomas sugestivos e a definição do diagnóstico real.

Este estudo apresenta limitações quanto a abordagem apenas dos Estados e capitais, sem a inclusão de dados sobre os municípios de origem dos casos confirmados; dados climáticos completos no período estudado e tamanho da população de animais envolvidos na cadeia de transmissão. A não realização de testes de estatísticos impediu a confirmação de algumas hipóteses surgidas ao término do estudo.

\section{CONCLUSÃO}

O número populacional de casos de leptospirose oscilou ao longo de todo período analisado, tendo assimetria de picos entre as regiões e capitais brasileiras. Predominou o sexo masculino, principalmente entre os $20 \mathrm{e}$ 39 anos, cor branca, baixa escolaridade, com infecção ocorrida em zona urbana, no próprio ambiente domiciliar e a maioria com desfecho de cura.

No território nacional não se conhece indícios de subnotificações de casos de leptospirose, porém diante do panorama atual, verifica-se a necessidade de maior atenção dos profissionais e gestores aos fatores de risco que possuem relação direta com o aumento da incidência da doença. Não obstante, isso requer novos estudos para melhor elucidação dos determinantes sociais de saúde e desiquilíbrios ambientais nas diversas regiões do país, proporcionando uma visão mais clara do comportamento da doença e orientando ações em saúde coletiva mais assertivas, capazes de modificar o cenário existente.

\section{REFERÊNCIAS}

1. Magalhaes VS, Acosta LMW. Leptospirose humana em Porto Alegre, Rio Grande do Sul, de 2007 a 2013: caracterização dos casos confirmados e distribuição espacial. Epidemiol Serv Saúde. 2019; 28(2):e2018192.

2. Chideroli RT, Gonçalves DD, Suphoronski AS, Alfieri AF, Alfieri AA, Oliveira AG, et al. Culture Strategies for Isolation of Fastidious Leptospira Serovar Hardjo and Molecular 
Differentiation of Genotypes Hardjobovis and Hardjoprajitno. Front microbiol. 2017; 8:2155.

3. Lara MCCSH, Villalobos EMC, Cunha SEM, Oliveira JV, Castro V, Nassar AFC. Occurrence of antibodies against Leptospira spp. in donkeys of São Paulo state. Arq Inst Biol. 2019; 86:e0582018.

4. Pinto-Ferreira F, Pasquali AKS, Thomas-Soccol V, Mitsuka-Breganó R, Caldart ET, Leandro AS, et al. Epidemiological relevance of dogs for the prevention of Toxoplasma gondii, Neospora caninum and Leptospira spp. Rev brap Parasitol vet. 2019; 3(28):383-394.

5. Martins MHM, Spink MJP. A leptospirose humana como doença duplamente negligenciada no Brasil. Ciênc Saúde colet. 2020; 3(25):919928.

6. Santos CVB, Mathias LA, Feitosa PJS, Oliveira JMB, Pinheiro JJW, Brandespim DF. Risk factors associated with leptospirosis in swine in state of Pernambuco, Brazil. Arq Inst Biol. 2019; 86:e0632017.

7. Souza IPO, Uberti MS, Tassinari WS. Geoprocessamento e análise espacial para identificação de áreas de risco para leptospirose: uma revisão sistemática. Rev inst med trop. 2020; $35(62)$.

8. Rodrigues CM. O círculo vicioso da negligência da leptospirose no Brasil. Rev Inst Adolfo Lutz. 2017; 76(único):1-11.

9. Schneider MC, Leonel DG, Hamrick PN, Caldas EP, Velasquez RT, Paez FAM, et al. Leptospirose na América Latina: explorando 0 primeiro conjunto de dados regionais. Rev panam saúde pública. 2017; 41.

10. Mottola C, Alho AM, Rafael T, Gonçalves T, Seixas R. Leptospirose em Portugal: Situação actual e importância das medidas de controlo no contexto da Saúde Pública. Rev electrónica vet. 2015; 16(4).

11. Scialfa E, Bolpe J, Bardón JC, Ridao G, Gentile J, Gallicchi O. Isolation of Leptospira interrogans from suburban rats in Tandil, Buenos Aires, Argentina. Rev argent microbiol. 2010; 42(2):126-8.

12. Oliveira DSC, Guimarães MBJ, Medeiros Z. Modelo Produtivo Para Leptospirose. Rev patol trop. 2009; $38(1): 17-26$.

13. Pavan MA, Cairó F, Pettinari MMJ, Samartino L, Brihuega B. Genotyping of Leptospira interrogans strains from Argentina by Multiple-Locus Variable-number tandem repeat 
Analysis (MLVA). Comp Immunol Microbiol Infect Dis. 2011; 34(2):13541.

14. Ataya GE, Bernal AMP, Vargas DC, Romero LY, Bernal CPJ, Castellanos NAM. Anticorpos anti-Leptospira spp. em distribuidores de carne na cidade de Tunja, Boyacá, Colômbia. J Bras Patol Med Lab. 2019; 55(2):122-135.

15. Instituto Brasileiro de Geografia e Estatística (IBGE). População estimada (Brasil). Rio de Janeiro: IBGE. [internet] 2019 [acesso em 2020 jul 01]. Disponível em: https://www.ibge.gov.br/cidades-eestados.html?view=municipio

16. Souza EP, Oliveira WTGH, Santos JPC, Toledo JP, Ferreira IPS, Esashika SNGS, et al. Doenças infecciosas e parasitárias no Brasil de 2010 a 2017: aspectos para vigilância em saúde. Rev panam saúde pública. 2020; 44:e10.

17. Cerveira RA, Ferreira LO, Oliveira EF, Felipe HKS, Almeida MCA, Lima SS, et al. Análise espaço-temporal da leptospirose na Amazônia Oriental, Estado do Pará, Brasil. Rev bras epidemiol. 2020; 23:e200041.

18. Chaiblich JV, Lima MLS, Oliveira RF, Monken M, Penna MLF. Estudo espacial de riscos à leptospirose no município do Rio de Janeiro (RJ). Saúde debate. 2017; (41):225-240.

19. Gonçalves NV, Araújo EM, Souza JAS, Pereira WMM, Miranda CSC, Campos PSS. Distribuição espaço-temporal da leptospirose e fatores de risco em Belém, Pará, Brasil. Ciênc saúde colet. 2016; 12(21):3947-3955.

20. Petrakovsky J, Bianchi A, Fisun $H$, Nájera-Aguilar P, Pereira MM. Animal Leptospirosis in Latin America and the Caribbean countries: Reported outbreaks and literature review (2002-2014). Int $j$ environ res public health (Online). 2014; 11(10):1077010789.

21. Silva AEP, Conceição GMS, Chiaravalloti Neto F. Spatial analysis and factors associated with leptospirosis in Santa Catarina, Brazil, 2001-2015. Rev Soc Bras Med Trop. 2020; 53:e20200466.

22. Núñez-González S, Gault C, Granja P, Simancas-Racines D. Spatial patterns of leptospirosis in Ecuador, 2013-2018. Trans R Soc Trop Med Hyg. 2020; 114(7):545-548.

23. Gutierrez JD. Effects of meteorological factors on human leptospirosis in Colombia. Int $\mathrm{J}$ Biometeorol. 2021; 65(2):257-263.

24. Oliveira HH, Rodrigues MAM, Santos IS, Francischetti CN. Perfil 
epidemiológico e socioeconômico da ocorrência de casos de leptospirose em municípios da Baixada Fluminense, Rio de Janeiro, Brasil. Enciclopédia Biosfera. 2016; 13(23):1479-1491.

25. Rodrigues AL. Perfil epidemiológico de pacientes acometidos por leptospirose em um estado brasileiro na Amazônia Ocidental. Rev SUSTINERE. 2019; 7(1):32-45.

26. Calado EJR, Oliveira VS, Dias FCF, Lopes AB, Oliveira AA, Santana VMX, et al. Leptospirose na região norte do Brasil: uma revisão da literatura e perfil epidemiológico comparativo.
Rev Patol Tocantins. 2017; 4(2):6571.

27. Threeswaran R, De Silva NL, Gooneratne L, Arya R, Handunnetti SM, Rajapakse S. Hemostatic derangement in leptospirosis: A prospective cross-sectional study. Int J Crit Illn Inj Sci. 2020; 10(Suppl 1):28-32.

28. Munoz-Zanzi C, Groene E, Morawski BM, Bonner K, Costa F, Bertherat E, et al. A systematic literature review of leptospirosis outbreaks worldwide, 1970-2012. Rev Panam Salud Publica. 2020; 44:e78.

Financiamento: Os autores declaram que não houve financiamento.

Conflito de interesses: Os autores declaram não haver conflito de interesses.

Participação dos autores:

- Concepção: Magalhães FAC, Mendes RM, Melo ALT.

- Desenvolvimento: Magalhães FAC, Mendes RM, Melo ALT.

- Redação e revisão: Magalhães FAC, Mendes RM, Melo ALT.

Como citar este artigo: Magalhães FAC, Mendes RM, Melo ALT. Análise descritiva dos casos confirmados de leptospirose em humanos no Brasil, período de 2010-2019. J Health NPEPS. 2021; 6(1):232-243.

Submissão: 06/07/2020

Aceito: $02 / 05 / 2021$

Publicado: 01/06/2021 